\title{
Single event-related changes in cerebral oxygenated hemoglobin using word game in schizophrenia
}

This article was published in the following Dove Press journal:

Neuropsychiatric Disease and Treatment

II December 2014

Number of times this article has been viewed

\author{
Ryo Fujiki ${ }^{1,2}$ \\ Kiichiro Morita ${ }^{1,2}$ \\ Mamoru Sato ${ }^{1,2}$ \\ Yuji Yamashita ${ }^{1,2}$ \\ Yusuke Kato ${ }^{1,2}$ \\ Yohei Ishii \\ Yoshihisa Shoji ${ }^{1,2}$ \\ Naohisa Uchimura' \\ 'Department of Neuropsychiatry, \\ Kurume University, ${ }^{2}$ Cognitive and \\ Molecular Research Institute of Brain \\ Diseases, Kurume University School \\ of Medicine, Kurume-City, Japan
}

\begin{abstract}
Neuroimaging studies have been conducted using word generation tasks and have shown greater hypofrontality in patients with schizophrenia compared with healthy subjects. In this study, we compared the characteristics of oxygenated hemoglobin changes involved in both phonological and categorical verbal fluency between 35 outpatients with schizophrenia and 35 healthy subjects during a Japanese "shiritori" task using single-event-related near-infrared spectroscopy. During this task, the schizophrenic patients showed significantly smaller activation in the prefrontal cortex area than the controls. In addition, a significant positive correlation was obtained between oxygenated hemoglobin changes (prefrontal cortex area, inferior parietal area) and the severity of positive psychiatric symptoms. It is possible that hypofrontality of patients may be a diagnostic assistance tool for schizophrenia, and that the relationship between activation and positive syndrome scores may be of help in predicting functional outcome in patients.
\end{abstract}

Keywords: word production task, single-event-related near-infrared spectroscopy, schizophrenia, hypofrontality, biological marker

\section{Introduction}

A number of neuroimaging studies have been conducted using word generation tasks and have reported greater frontal hypoactivity in patients with schizophrenia compared with healthy subjects. ${ }^{1-3}$ Shiritori is a very popular word game in Japan and it is possible to play from 3 years old or more. ${ }^{4}$ This game requires players to generate a noun that begins with the last character of the noun given by the preceding player, necessitating players to retain information about characters and manipulate information - that is, working memory.

Near-infrared spectroscopy (NIRS), which estimates brain activity by measuring relative changes in oxygenated hemoglobin (oxy- $\mathrm{Hb}$ ) concentration at the surface of the brain, has increasingly been performed for several neuropsychological tests of brain functions. Advantages of this apparatus are the lack of sensitivity to body movement; it's relatively small size, portability, and inexpensiveness as compared to other neuroimaging settings; ${ }^{1,2}$ and it enables the researcher to create a mapping of the brain's functional activities; thus, this machine is possibly a useful tool for assessing cortical activation patterns of patients with schizophrenia during shiritori performance. To our knowledge, previous findings have not been reported regarding the cortical activation between patients with schizophrenia and healthy subjects during a shiritori task using NIRS; however, there have been functional magnetic resonance imaging (MRI) and magnetoencephalography studies using this task in only healthy subjects..$^{4,5}$
Correspondence: Ryo Fujiki and Cognitive and Molecular Research Institute of Brain Diseases, Kurume University School of Medicine 67, Asahi-Machi, Kurume-City, Japan Tel +81942317564

Fax +8I 94235604 I

Email fujiki_ryou@med.kurume-u.ac.jp 
The present study investigated single-event-related changes in cortical activation during a shiritori task in healthy subjects and patients with schizophrenia by using a multichannel NIRS machine.

The purposes of this study were to investigate different activity patterns in patients with schizophrenia compared with those in healthy subjects and to find the relationship between psychiatric symptoms and functional brain activities. We assumed that relative frontal hypoactivation would be found in schizophrenic patients compared to healthy subjects and that an association between activation and symptom scores could be an indicator of clinical outcome, as in previous studies.

\section{Materials and methods Participants}

This study enrolled 35 outpatients with schizophrenia (19 males and 16 females; mean age, 29.4 \pm 5.8 years, paranoid type) and the same number of healthy control subjects (19 males and 16 females; mean age, $27.6 \pm 6.8$ years). In accordance with the International Classification of Disease, tenth revision, two attending psychiatrists diagnosed patients. ${ }^{6}$ All participants were native Japanese speakers and were judged from the Edinburgh Inventory to be righthanded. ${ }^{7}$ The Japanese version of the National Adult Reading Test assessed their mean intelligence quotient (IQ) values. ${ }^{8}$ No subjects had a head injury, neurologic disorder, alcohol/ substance abuse, epilepsy, visual disabilities, aphasia, or dyslexia. The healthy subjects had no history of psychiatric disorders or familial history of psychosis. The schizophrenic patients were taking atypical antipsychotic medications (ie, risperidone [ $n=19]$, olanzapine [ $n=12]$, and quetiapine [ $n=4]$ ) and were assessed by the Positive and Negative Syndrome Scale (PANSS). ${ }^{9}$ All of them were engaged in a community workshop and/or housework. The mean daily antipsychotic dose in terms of haloperidol equivalent was $7.5 \pm 1.8 \mathrm{mg} .{ }^{10}$ Adequate informed consent was obtained from all subjects. This study was approved by the ethics committee of Kurume University. Demographic and clinical characteristics of all subjects are shown in Table 1.

\section{Procedure}

\section{NIRS measurement}

A 44-channel NIRS system (ETG4000; Hitachi, Tokyo, Japan) measured oxy-Hb changes during tasks covering from the frontal to temporoparietal regions. Oxy-Hb changes were calculated from the difference in absorbance based on the modified Beer-Lambert law. The middle point of
Table I Subject characteristics

\begin{tabular}{llll}
\hline & Patients & Controls & $P$-value \\
\hline Age (year) & $29.4 \pm 5.8$ & $27.6 \pm 6.8$ & 0.25 \\
Sex (F/M) & $19 / 16$ & $19 / 16$ & \\
Education (year) & $13.9 \pm 1.3$ & $15.7 \pm 1.6$ & $<0.05$ \\
IQ & $100.3 \pm 8.1$ & $103.2 \pm 5.3$ & 0.08 \\
Illness duration (year) & $4.2 \pm 1.9$ & & \\
PANSS score & & & \\
$\quad$ Positive subscale & $18.2 \pm 4.9$ & & \\
$\quad$ Negative subscale & $19.9 \pm 3.6$ & & \\
$\quad$ General score & $41.0 \pm 4.3$ & & \\
Medication & & & \\
$\quad$ HPD equivalent (mg/day) & $7.5 \pm 1.8$ & & \\
\hline
\end{tabular}

Note: Values are expressed as mean \pm standard deviation.

Abbreviations: F, female; HPD, haloperidol; IQ, intelligence quotient; M, male; PANSS, Positive and Negative Syndrome Scale.

the injector-detector probe pairs was defined as a channel. The depth of each channel is supposed to measure changes at points 2 to $3 \mathrm{~cm}$ from the scalp that correspond to the cerebral cortical surface. ${ }^{11,12}$ According to the International 10-20 system used in electroencephalography, we placed probes along the Fp1-Fp2 line to the lowest anterior probes; left channel 19 and right channel 22 (Figure 1A). To avoid movement artifacts, participants were instructed to minimize their movements and jaw fixation during examination. The pre-task baseline was determined as the mean during 1 second preceding the word presented, while the post-task baseline was determined as the mean during 1 second from 10 to 11 seconds after the word was presented. Linear fitting was applied to the data between these two baselines. For the relationship between each channel and anatomic region, NIRS data were converted to a normalized brain image template (three-dimensional composition indication unit; Hitachi).

\section{Task design}

Brain activation was measured during word production. The advantage of NIRS is that it is relatively insensitive to body movements during measurement; therefore, this apparatus can obtain data in an overt task. For this examination, each subject sat on a comfortable chair and was required to perform word production. One session consisted of two contrasting conditions (word production task, control condition), and all subjects alternated between these conditions. Each word was visually presented by a monitor for 0.3 seconds as an activation task and a fixed circle was presented for 12 seconds. In the activation task, subjects had to immediately generate a noun that starts with the last kana character of the presented word and they were required to say only animal nouns. Thus, this task was the animal category version of the Japanese shiritori word game, as well as a word production task. For example, 
A

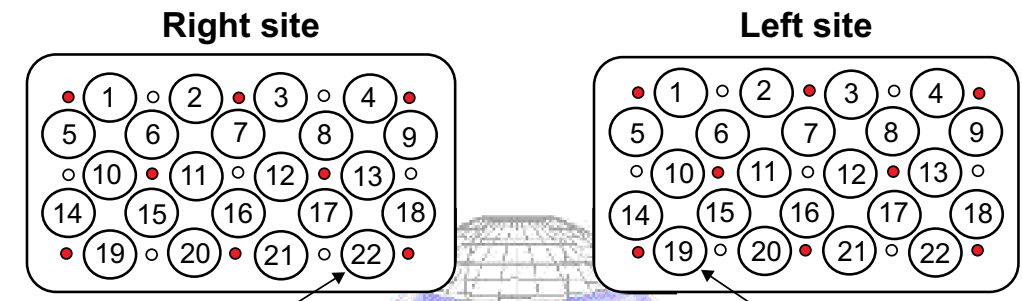

Fp-2

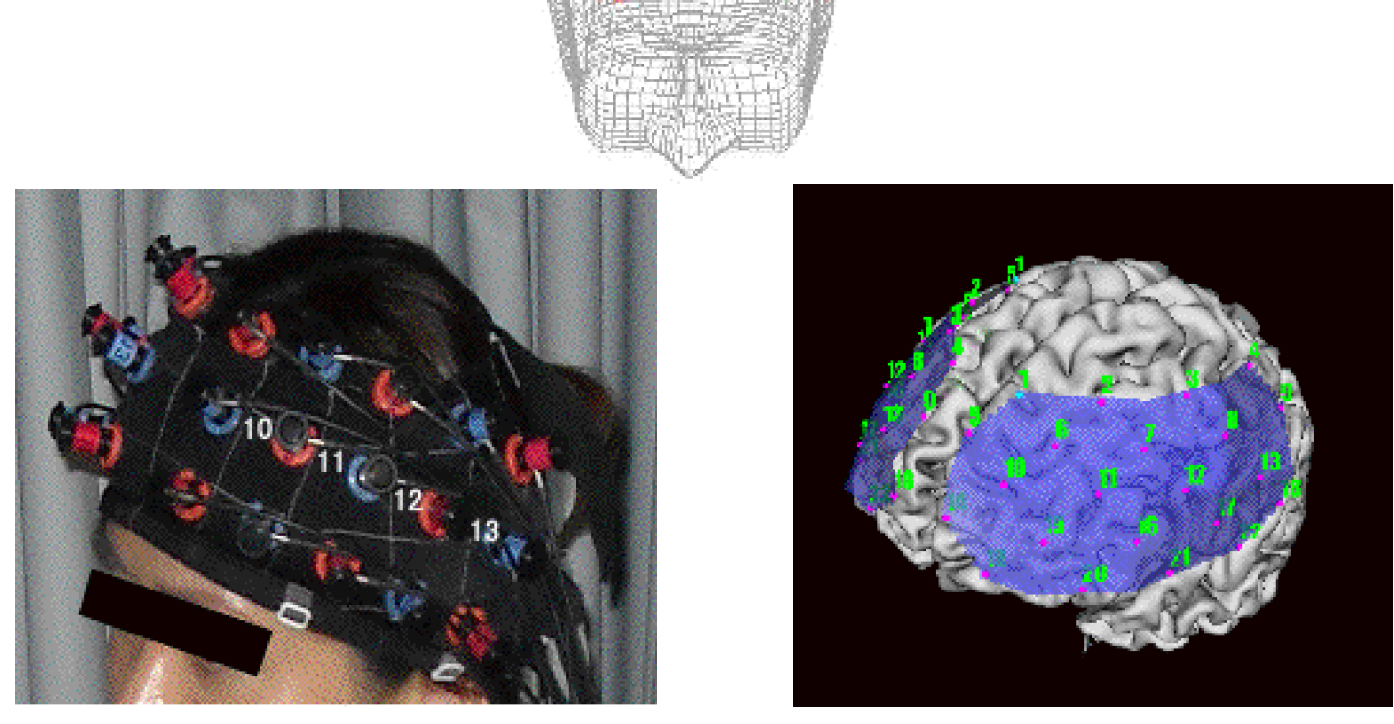

Fp-1
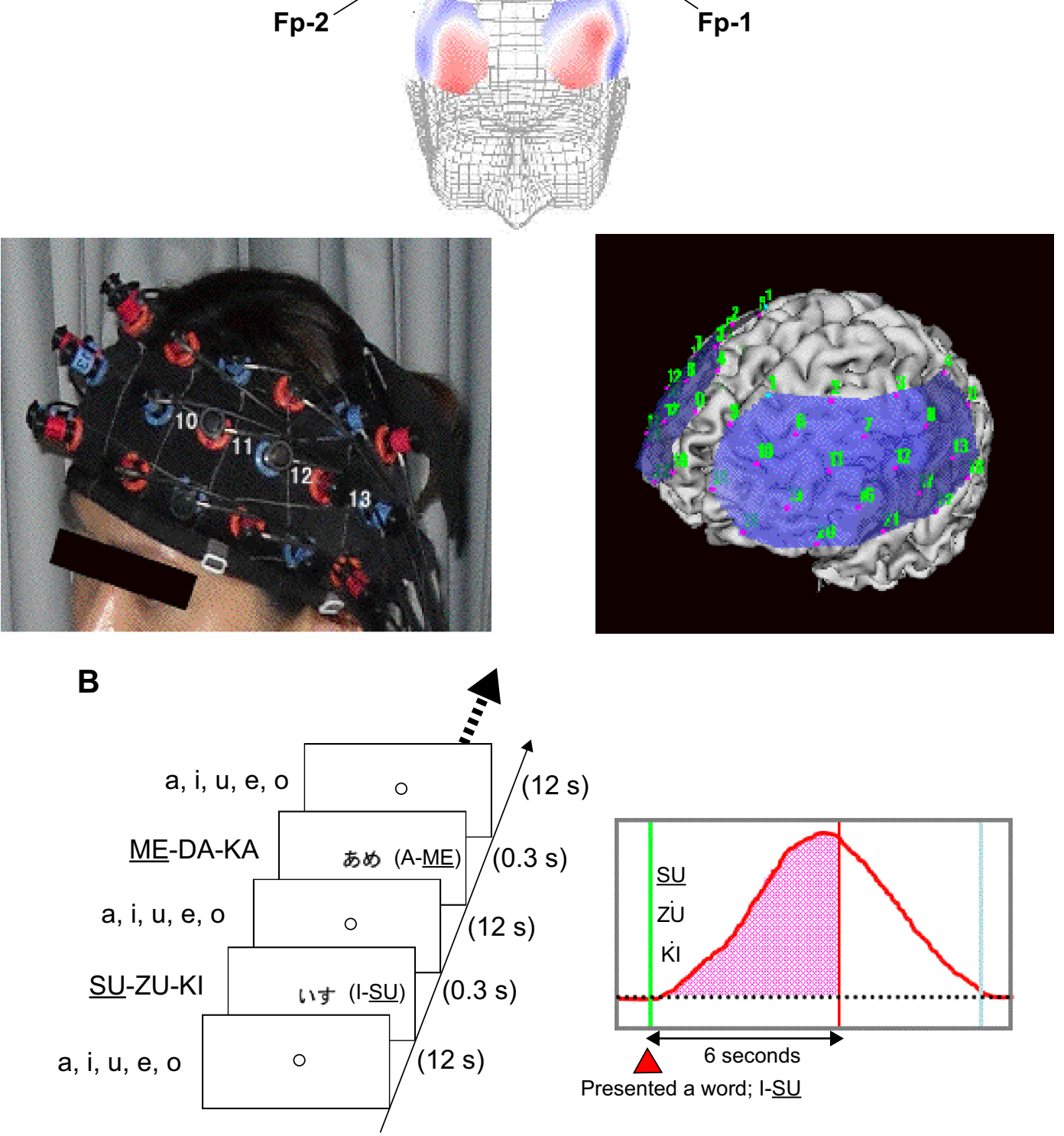

\section{Fp-1}

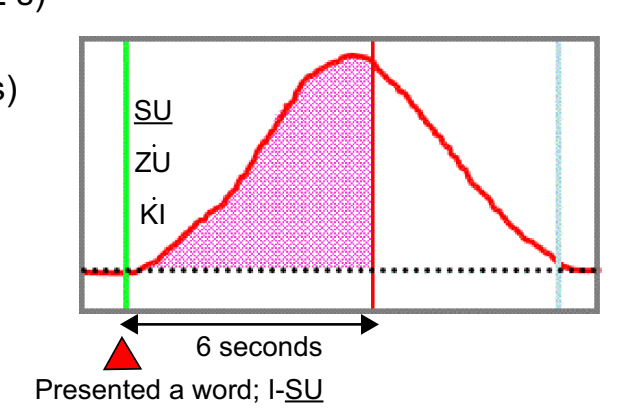

Figure I Study design: (A) location of channels, (B) protocol.

Notes: (A) Subjects were instructed to generate a noun for a presented word. This procedure was repeated 20-25 times. Subjects had to generate a noun-only creature. This task is the creature category and letter version of the verbal shiritori task. (B) Data were calculated from the mean wave of oxy-HB changes. The area of the waveform of oxy-HB changes 6 seconds after the presented word was calculated and we used values of this area as NIRS data.

Abbreviations: NIRS, near-infrared spectroscopy; oxy-HB, oxygenated hemoglobin; s, seconds.

when the noun "I-SU" (chair) was presented, the subject said the noun "SU-ZU-KI" (sea bass). In the control condition, subjects were required to say the syllables "A-I-U-E-O" repeatedly. The word production task was repeated 20-25 times per session. Waveforms of 20 correct responses for each subject were adopted as law data, and we discarded waveforms of incorrect responses and responses over 1 second after the presented task. Averaged waveforms for the 20 correct responses were analyzed using the "integral mode" of the apparatus. Oxy-Hb changes between activation and control periods were expressed numerically for every 100 milliseconds. NIRS data were calculated from the mean wave of oxy-Hb changes. 
The area of the waveform 6 seconds after the presented word was calculated because peak latency of healthy subjects was $5.0 \pm 0.9$ seconds during this task in a pilot study, and we used values of this area as NIRS data (Figure 1B).

\section{Statistical analysis}

Raw NIRS data were preprocessed by applying a low pass filter with cutoff frequencies of $0.5 \mathrm{~Hz}$. We used histograms at each channel to confirm a normal distribution. Performance and oxy-Hb changes at each channel in controls were compared with those of patients with schizophrenia using an unpaired $t$-test. For analyzing cortical activation, we adopted the false discovery rate correction method (Benjamini and Hockberg method) $)^{13}$ and set the value specifying the maximum false discovery rate to 0.05 . Next, to evaluate the relationship between activation and the PANSS score/ profile, the Pearson's product-moment correlation coefficient was calculated. For $-0.4<r<0.4$, it is considered to be a low correlation (not very significant); correlation coefficients were accepted as significant at $r>0.4$ and $r<-0.4$. $P<0.05$. The averaged values were expressed as mean \pm standard deviation. Strangman et al reported that oxy-Hb changes correlate more strongly with blood-oxygen-level dependent (BOLD) functional MRI signal than do deoxygenated hemoglobin changes; therefore, we adopted the oxy-Hb changes as activation data. ${ }^{14}$

\section{Results}

\section{Performance of word production task}

Word production was $21.1 \pm 1.8$ words in the schizophrenic patients and $21.5 \pm 0.9$ words in the healthy controls. There was no significant difference between the groups (unpaired $t$-test: $t=1.6, P=0.120)$.

\section{Activation during word production task at each channel}

The healthy subjects had significantly larger activation than the patients with schizophrenia at five channels (left channel 2, unpaired $t$-test: $t=3.0$, degrees of freedom $[d f]=68$, $q=0.004$; left channel 11, unpaired $t$-test: $t=3.5, d f=68$, $q=0.001$; left channel 12, unpaired $t$-test: $t=3.0, d f=68$, $q=0.003$; left channel 19, unpaired $t$-test: $t=3.6, d f=68$, $q<0.001$; and right channel 17, unpaired $t$-test: $t=3.1, d f=68$, $q=0.003 ; q$-value $=0.001-0.006)$ (Figure 2$)$. Waveforms are shown in Figure 3.

\section{Correlation between oxy-Hb changes and PANSS scores or performance}

Oxy-Hb changes at left channel four, left channel seven, left channel ten, left channel eleven, right channel one, right channel eight, and right channel 13 revealed a significant positive correlation with positive symptom scores in patients ( $r=0.45, P=0.003 ; r=0.41, P=0.007 ; r=0.48, P=0.002 ; r=0.51$, $P=0.001 ; r=0.44, P=0.006 ; r=0.41, P=0.008$; and $r=0.44$, $P=0.005$, respectively); however, there was no correlation with negative symptom scores (Figure 4). No correlation was found between performance and activation in both groups. In the schizophrenic patients, oxy-Hb changes at each channel showed no significant correlation with illness duration and antipsychotic drug dose. We investigated the relationship between activation and each drug, and did not find any statistical significance. There was no significant correlation

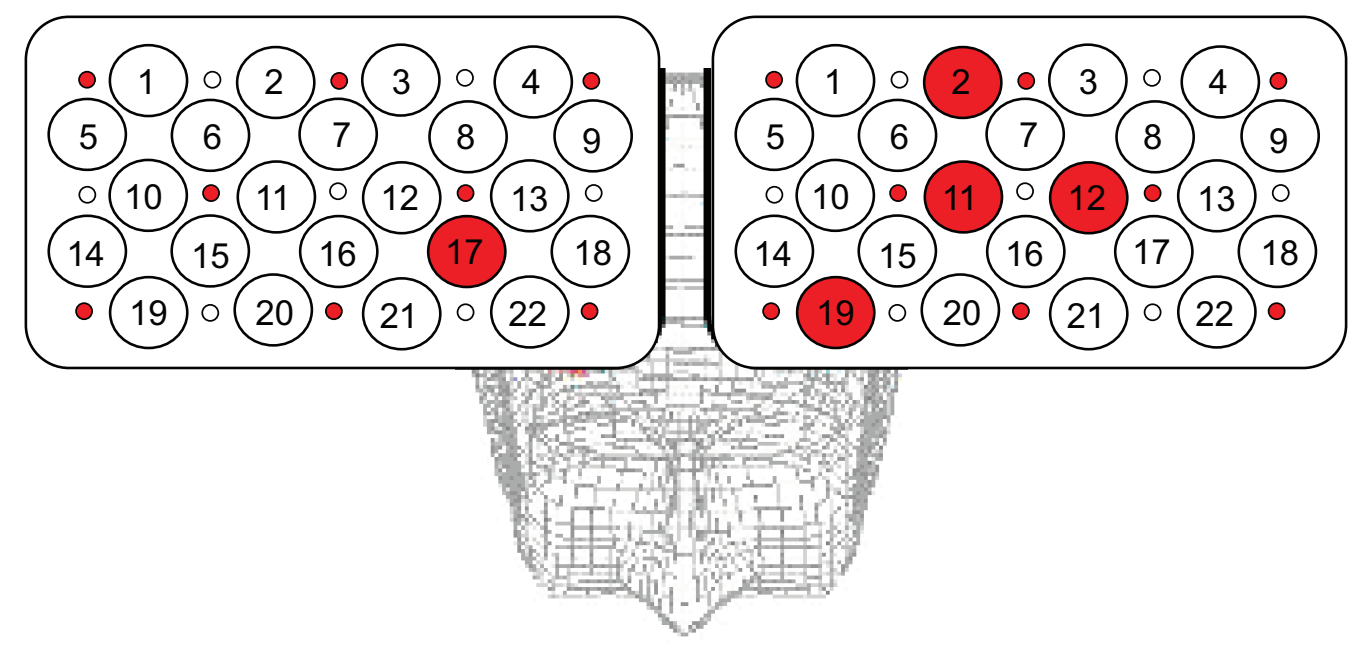

Figure 2 Maps of cortical distribution of channels that were significantly smaller in patients than in healthy subjects. Note: Statistically significant channels are colored red. 

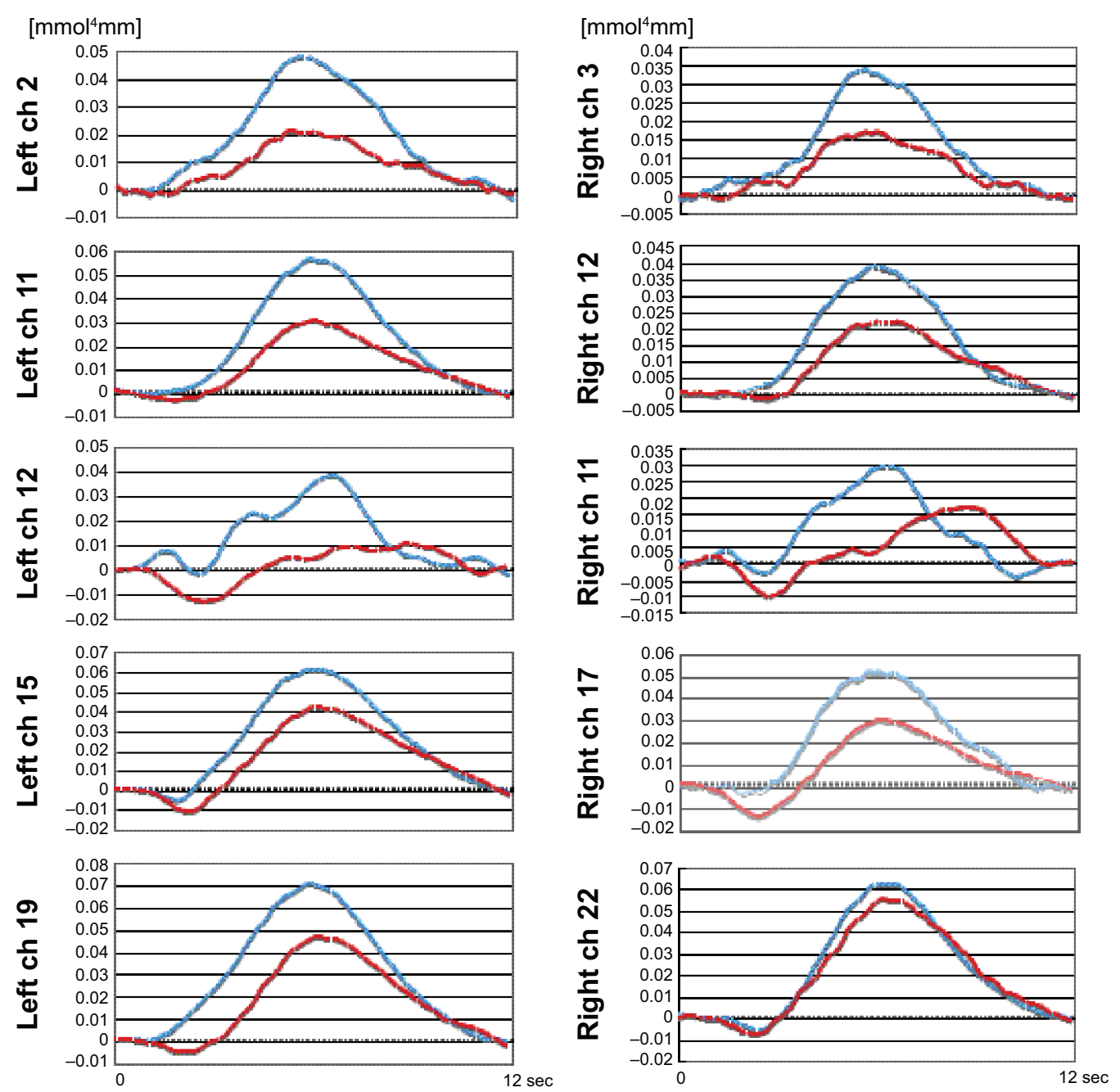

Figure 3 Waveforms in healthy subjects and patients at significantly different activation channels.

Note: Blue lines are healthy subjects and red lines are patients.

Abbreviation: ch, channel.

between performance and PANSS scores or IQ. In addition, there was no significant correlation between medication and PANSS scores or IQ.

\section{Discussion}

We compared hemodynamic changes between patients with schizophrenia and healthy subjects during a word production task using a single-event-related NIRS. In addition, we investigated the relationship between regional activation and PANSS scores in patients. A previous study suggested that NIRS measurement using an event-related method enables researchers to obtain more efficient averaging and to be free from artifacts. ${ }^{15}$ Thus, we thought that this task of performing overt word production would enable the evaluation of psychiatric patients. This task is associated with the characteristics of both letter and categorical verbal fluency and requires several cognitive functions, such as retrieval, attention, and working memory. In Japanese, Sumiyoshi et al showed no difference in degree of impairments between the letter and category version of this task. ${ }^{16}$ The present study showed pronounced hypoactivation of the prefrontal cortex (PFC) area in the schizophrenic patients compared to the healthy subjects as in other studies, ${ }^{1,2,17-20}$ while it has been reported that hyperactivation is a feature of the schizophrenic patients. ${ }^{21}$ Manoach suggested that both findings reflected PFC dysfunction in schizophrenia, rather than that one of them explained the characteristics of inefficiency in patients. ${ }^{21}$ Van Snellenberg et al reported that the relationship between an increase in load on working memory and PFC activation showed an inversed U-curve: ${ }^{22}$ PFC activation was enhanced as the load increased, but then declined after exceeding a specific level even in healthy subjects. ${ }^{20,22}$ In patients with schizophrenia, this curve may shift leftward and reach a peak in the presence of a small load. The presence 


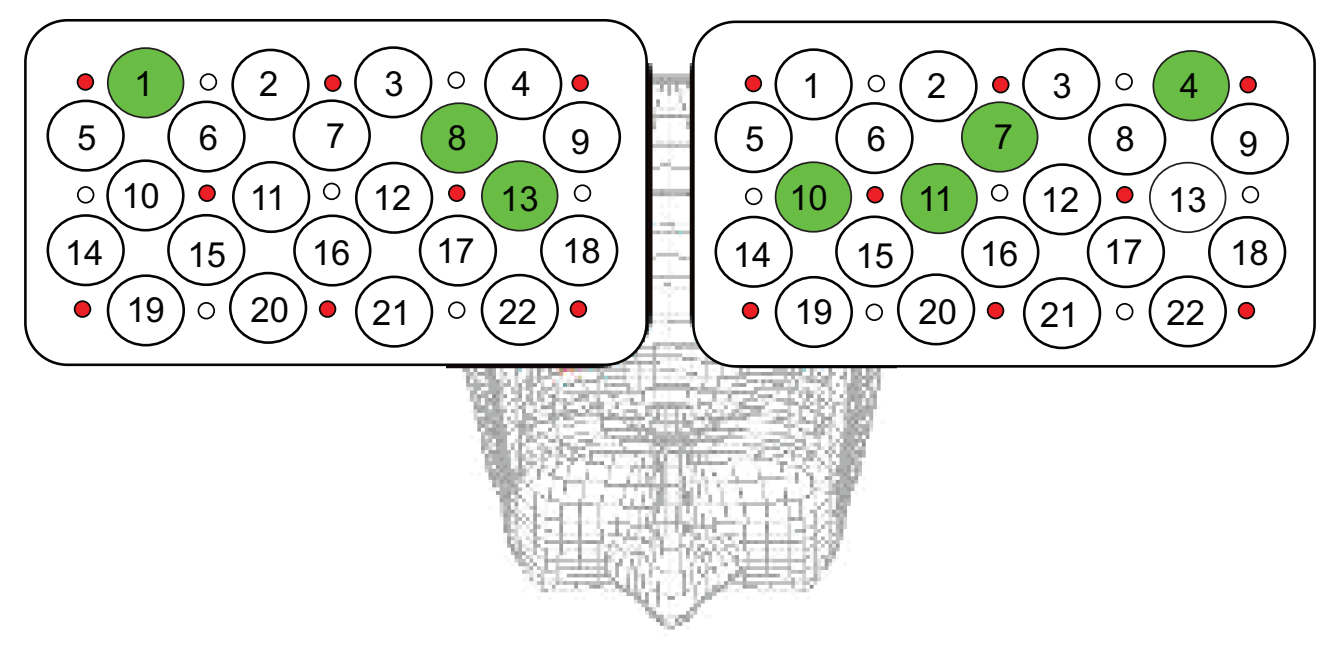

Figure 4 Cortical distribution of significant correlations between activation and PANSS scores.

Note: Statistically significant channels correlated with positive symptom scores are colored green.

Abbreviation: PANSS, Positive and Negative Syndrome Scale.

of hypofrontality in schizophrenic patients was likely to be associated with the difference in the difficulty level between the groups even when performing the same task. According to the waveforms presented at each channel, it seemed that the difference between the groups showed in the amplitude rather than the latency of waveform, although we could not test this exactly. We thought that the hypofrontality of the schizophrenic patients was influenced by a lesser degree of activation rather than a more delayed activation. Further study is needed to examine these points in detail.

In short, it is suggested that a heavy load was imposed on the schizophrenia patients compared to the healthy subjects, although task performance showed no significant differences between groups. However, there are some issues regarding hyperfrontality/hypofrontality that have to be mentioned. Schizophrenia has been associated with deficits in attention, executive processes, and working memory; ${ }^{23}$ therefore, the reduced activity of the schizophrenic patients might have been due to decreased motivation, fatigue, or impaired attention specific to the disease. Taking these issues into account, we ensured that the number of task executions for one session was less than the event-related potential of electroencephalography, and the total time of an examination for each subject was less than 5 minutes.

We investigated the relationship between functional brain activity and PANSS scores. Previous studies have demonstrated a significant correlation between PFC activation and psychiatric symptoms. ${ }^{18,24-26}$ In the present study, there was a correlation not only with the PFC area (left channel $7,10,11$; right channel 8,13 ) but also with the inferior parietal area (left channel 4, right channel 1). In working memory tasks such as the verbal fluency test, past studies have showed that the phonological loop is associated with the left inferiorparietal region and that the central executive system is associated with the prefrontal region. ${ }^{27}$ Thus, we suggest that these findings reflect the fact that PFC function was involved in the word production task and inferior parietal function was induced by phonological working memory. It is possible that the relationship between right inferiorparietal activation and PANSS positive subscale reflect inefficiency of schizophrenia; this finding may be the result of a compensation mechanism.

Our results suggest that cortical activation predicts the outcome of patients, as suggested by previous findings. ${ }^{18,24-26}$ Therefore, it is believed that hypofrontality of schizophrenic patients may be a trait marker, a view that is controversial, and the relationship between activation and PANSS scores may be a state marker in patients with schizophrenia. A previous study attempted to identify the specific psychiatric disease during the verbal fluency task using NIRS, but failed to prove that the method was a useful diagnostic tool. ${ }^{17}$ Indeed, hypoactivation of several psychiatric disorders, such as mood disorder, dementia, anxiety disorder, and eating disorder have been reported. ${ }^{28-35}$ Our findings on hypoactivation are limited to biological markers in a psychiatric disease, so further studies are needed to find disease-specific features.

There are some limitations to the present study. First, the spatial resolution of NIRS is lower than that of MRI. We converted our data to a normalized brain image template for the relationship between recording site and anatomic region 
(Figure 1A). In addition, we adopted the International 10-20 system for brain mapping as in a previous report. ${ }^{36}$ Second, regarding such artifacts as body movement and muscle activity, NIRS is relatively insensitive to body movement and can be performed in a natural posture, but artifacts are likely to be produced by nodding and rotation of the head. We therefore employed jaw fixation and data averaging using the event-related method, which is insensitive to noise because of low-frequency signal components, to reduce artifacts as much as possible. ${ }^{15}$ A previous study also investigated the relationship between frontotemporal muscle activity and NIRS signal, ${ }^{37}$ and found no significant correlations. Third, regarding medications, all patients received atypical antipsychotic drugs, with which cognitive impairment has been associated. ${ }^{38,39}$ However, cognitive impairment has been reported in untreated patients with schizophrenia, in patients about to begin treatment, and in patients at the clinical onset of schizophrenia, as well as in healthy first-degree relatives of patients with schizophrenia. ${ }^{40-43}$ In this study, there was no relationship between activation and each drug; however, future studies should investigate drug-naive patients. Fourth, regarding task performance, there were no differences in performance between groups. The present study investigated only waveforms of correct responses and only adopted data of responses of less than 1 second, but we did not measure reaction time. Fifth, although this study did not investigate the difference between oxy-Hb and deoxygenated hemoglobin, we adopted oxy-Hb changes as data similar to previous study ${ }^{14}$ because BOLD signal and NIRS signal has been investigated in the different types of blood vessels. Therefore, further studies are needed to examine this point in detail.

In summary, this study performed a single-event-related NIRS method during a shiritori game task involving characteristics of both letter and category verbal fluency in patients with schizophrenia versus healthy controls. We revealed reduced PFC activity and a significantly positive correlation between oxy-Hb changes and positive psychiatric symptom scores in patients with schizophrenia. It is possible that hypofrontality of patients is not a disease-related feature, and may be a diagnostic assistance tool for schizophrenia. In addition, an observed relationship between activation and PANSS scores may be of help in predicting functional outcome in patients.

\section{Acknowledgment}

This work was supported by a Japanese Grant-in-Aid for Science Research (20591425).

\section{Disclosure}

The authors report no conflicts of interest in this work.

\section{References}

1. Watanabe A, Kato T. Cerebrovascular response to cognitive tasks in patients with schizophrenia measured by near-infrared spectroscopy. Schizophr Bull. 2004;30(2):435-444.

2. Ehlis AC, Herrmann MJ, Plichta MM, Fallgatter AJ. Cortical activation during two verbal fluency tasks in schizophrenic patients and healthy controls as assessed by multi-channel near-infared spectroscopy. Psychiatry Res. 2007;156(1):1-13.

3. Walter H, Wunderlich AP, Blankenhorn M, et al. No hypofrontality, but absence of prefrontal lateralization comparing verbal and spatial working memory in schizophrenia. Schizophr Res. 2003;61(2-3):175-184.

4. Yamamoto M, Ukai S, Shinosaki K, et al. Spatially filtered magnetoencephalographic analysis of cortical oscillatory changes in basic brain rhythms during the Japanese 'Shiritori' Word Generation Task. Neuropsychobiology. 2006;53(4):215-222.

5. Inoue M, Ueno T, Morita K, et al. Brain activities on fMRI using shiritori task in normal subjects. Kurume Med J. 2011;57(4):109-115.

6. World Health Organization. The ICD-10 Classification of Mental and Behavioural Disorders: Diagnostic criteria for research. Geneva: World Health Organization; 1993. Available from: http://www.who.int/ classifications/icd/en/GRNBOOK.pdf. Accessed October 30, 2014.

7. Oldfield RC. The assessment and analysis of handedness: the Edinburgh inventory. Neuropsychologia. 1971;9(1):97-113.

8. Uetsuki M, Matsuoka K, Kim Y, et al. [Estimation of premorbid IQ by JART in schizophrenia]. Seishinigaku. 2006;48:15-22. Japanese.

9. Key SR, Opler LA, Fiszbein A (translated by Yamada H, Matsui K, Kikumoto K). [Positive and Negative Syndrome Scale (PANSS) Rating Manual]. Tokyo: Seiwa Shoten Publishers; 1991. Japanese.

10. Kane JM, Leucht S, Carpenter D, Docherty JP; Expert Consensus Panel for Optimizing Pharmacologic Treatment of Psychotic Disorders. The expert consensus guideline series. Optimizing pharmacologic treatment of psychotic disorders. Introduction: methods, commentary, and summary. J Clin Psychiatry. 2003;64 Suppl 12:5-19.

11. Okada E, Delpy DT. Near-infrared light propagation in an adult head model. I. Modeling of low-level scattering in the cerebrospinal fluid layer. Appl Opt. 2003;42(16):2906-2914.

12. Okada E, Delpy DT. Near-infrared light propagation in an adult head model. II. Effect of superficial tissue thickness on the sensitivity of the near-infrared spectroscopy signal. Appl Opt. 2003;42(16): 2915-2922.

13. Benjamini Y, Hockberg Y. Controlling the false discovery rate: a practical and powerful approach to multiple testing. $J$ R Stat Soc Series B Stat Methodol. 1995;57(1):289-300.

14. Strangman G, Culver JP, Thompson JH, Boas DA. A quantitative comparison of simultaneous BOLD fMRI and NIRS recordings during functional brain activation. Neuroimage. 2002;17(2):719-731.

15. Jasdzewski G, Strangman G, Wagner J, Kwong KK, Poldrack RA, Boas DA. Differences in the hemodynamic response to event-related motor and visual paradigms as measured by near-infrared spectroscopy. Neuroimage. 2003;20(1):479-488.

16. Sumiyoshi C, Sumiyoshi T, Matsui M, et al. Effect of orthography on the verbal fluency performance in schizophrenia: examination using Japanese patients. Schizophr Res. 2004;69(1):15-22.

17. Azechi M, Iwase M, Ikezawa K, et al. Discriminant analysis in schizophrenia and healthy subjects using prefrontal activation during frontal lobe tasks: a near-infrared spectroscopy. Schizophr Res. 2010;117(1): $52-60$.

18. Ikezawa K, Iwase M, Ishii R, et al. Impaired regional hemodynamic response in schizophrenia during multiple prefrontal activation tasks: a two-channel near-infrared spectroscopy study. Schizophr Res. 2009; 108(1-3):93-103. 
19. Suto T, Fukuda M, Ito M, Uehara T, Mikuni M. Multichannel near-infrared spectroscopy in depression and schizophrenia: cognitive brain activation study. Biol Psychiatry. 2004;55(5):501-511.

20. Carter CS, Perlstein W, Ganguli R, Brar J, Mintun M, Cohen JD. Functional hypofrontality and working memory dysfunction in schizophrenia. Am J Psychiatry. 1998;155(9):1285-1287.

21. Manoach DS. Prefrontal cortex dysfunction during working memory performance in schizophrenia: reconciling discrepant findings. Schizophr Res. 2003;60(2-3):285-298.

22. Van Snellenberg JX, Torres IJ, Thornton AE. Functional neuroimaging of working memory in schizophrenia: task performance as a moderating variable. Neuropsychology. 2006;20(5):497-510.

23. Baddeley A, Wilson B. Frontal amnesia and the dysexecutive syndrome. Brain Cogn. 1988;7(2):212-230.

24. Henseler I, Falkai P, Gruber O. A systematic fMRI investigation of the brain systems subserving different working memory components in schizophrenia. Eur J Neurosci. 2009;30(4):693-702.

25. Menon V, Anagnoson RT, Mathalon DH, Glover GH, Pfefferbaum A. Functional neuroanatomy of auditory working memory in schizophrenia: relation to positive and negative symptoms. Neuroimage. 2001; 13(3):433-446.

26. Fujiki R, Morita K, Sato M, et al. Reduced prefrontal cortex activation using the Trail Making Test in schizophrenia. Neuropsychiatr Dis Treat. 2013;9:675-685.

27. Baddeley A. Working memory: looking back and looking forward. Nat Rev Neurosci. 2003;4(10):829-839.

28. Herrmann M, Ehlis AC, Fallgatter AJ. Bilaterally reduced frontal activation during a verbal fluency task in depressed patients as measured by near-infrared spectroscopy. J Neuropsychiatry Clin Neurosci. 2004; 16(2):170-175.

29. Matsuo K, Kouno T, Hatch JP, et al. A near-infrared spectroscopy study of prefrontal cortex activation during a verbal fluency task and carbon dioxide inhalation in individuals with bipolar disorder. Bipolar Disord. 2007; 9(8):876-883.

30. Pu S, Matsumura H, Yamada T, et al. Reduced frontopolar activation during verbal fluency task associated with poor social functioning in late-onset major depression: Multi-channel near-infrared spectroscopy study. Psychiatry Clin Neurosci. 2008;62(6):728-737.

31. AraiH, Takano M, MiyakawaK, etal. A quantitative near-infrared spectroscopy study: a decrease in cerebral hemoglobin oxygenation in Alzheimer's disease and mild cognitive impairment. Brain Cogn. 2006;61(2): 189-194.
32. Herrmann MJ, Langer JB, Jacob C, Ehlis AC, Fallgatter AJ. Reduced prefrontal oxygenation in Alzheimer disease during verbal fluency tasks. Am J Geriatr Psychiatry. 2008;16(2):125-135.

33. Nishimura Y, Tanii H, Fukuda M, et al. Frontal dysfunction during a cognitive task in drug-naive patients with panic disorder as investigated by multi-channel near-infrared spectroscopy imaging. Neurosci Res. 2007;59(1):107-112.

34. Ohta H, Yamagata B, Tomioka H, et al. Hypofrontality in panic disorder and major depressive disorder assessed by multi-channel near-infrared spectroscopy. Depress Anxiety. 2008;25(12):1053-1059.

35. Uehara T, Fukuda M, Suda M, et al. Cerebral blood volume changes in patients with eating disorders during word fluency: a preliminary study using multi-channel near infrared spectroscopy. Eat Weight Disord. 2007;12(4):183-190.

36. Okamoto M, Dan H, Sakamoto K, et al. Three-dimensional probabilistic anatomical cranio-cerebral correlation via the international 10-20 system oriented for transcranial functional brain mapping. Neuroimage. 2004;21(1):99-111.

37. Schecklmann M, Ehlis AC, Plichta MM, Fallgatter AJ. Influence of muscle activity on brain oxygenation during verbal fluency assessed with functional near-infrared spectroscopy. Neuroscience. 2010; 171(2):434-442.

38. McGurk SR, Carter C, Goldman R, et al. The effects of clozapine and risperidone on spatial working memory in schizophrenia. Am J Psychiatry. 2005;162(5):1013-1016.

39. Reilly JL, Harris MS, Keshavan MS, Sweeney JA. Adverse effects of risperidone on spatial working memory in first-episode schizophrenia. Arch Gen Psychiatry. 2006;63(11):1189-1197.

40. Zabala A, Rapado M, Arango C, et al. Neuropsychological functioning in early-onset first-episode psychosis: comparison of diagnostic subgroups. Eur Arch Psychiatry Clin Neurosci. 2010;260(3):225-233.

41. Park S, Holzman PS, Goldman-Rakic PS. Spatial working memory deficits in the relatives of schizophrenic patients. Arch Gen Psychiatry. 1995;52(10):821-828.

42. Myles-Worsley M, Park S. Spatial working memory deficits in schizophrenia patients and their first degree relatives from Palau, Micronesia. Am J Med Genet. 2002;114(6):609-615.

43. Bowie PD, Harvey PB. Cognitive deficits and functional outcome in schizophrenia. Neuropsychiatr Dis Treat. 2006;2(4):531-536.
Neuropsychiatric Disease and Treatment

\section{Publish your work in this journal}

Neuropsychiatric Disease and Treatment is an international, peerreviewed journal of clinical therapeutics and pharmacology focusing on concise rapid reporting of clinical or pre-clinical studies on a range of neuropsychiatric and neurological disorders. This journal is indexed on PubMed Central, the 'PsycINFO' database and CAS,

\section{Dovepress}

and is the official journal of The International Neuropsychiatric Association (INA). The manuscript management system is completely online and includes a very quick and fair peer-review system, which is all easy to use. Visit http://www.dovepress.com/testimonials.php to read real quotes from published authors. 